\title{
A Multithreaded Algorithm for Mining Maximal Cohesive Dense Modules from Interaction Networks with Gene Profiles
}

\author{
Aditya Goparaju \\ North Dakota State University \\ Fargo, North Dakota 58102 \\ aditya.goparaju@ndsu.edu
}

\author{
Saeed Salem \\ North Dakota State University \\ Fargo, North Dakota 58102 \\ saeed.salem@ndsu.edu
}

\begin{abstract}
Several graph datasets exist which have additional attributes, representing properties of either nodes or edges in the graph. Recent research has focused on finding integrated or cohesive clusters where the clusters are not only densely connected but also have similarities in a subspace of their attributes. Cohesive clusters are more robust and accurately represent the cluster structure as they exhibit similarity in two domains (network structure and attributes).

In this paper, we propose a multithreaded enumeration technique, for mining maximal cohesive and dense clusters from node-attributed graphs. For relaxed constraints the number of result clusters on a large graph can be very high and we need a way to find a representative set of clusters which can be most similar to all remaining clusters. We propose a novel technique to find representative clusters from the output space. Experiments on two real interaction networks show the effectiveness of the proposed algorithm.
\end{abstract}

\section{Categories and Subject Descriptors}

J.3 [Life and Medical Sciences]: - Biology and genetics; H.2.8 [Database Management]: Database Applications-Data mining, Scientific databases; H.3.3 [Information Storage and Retrieval]: Information Search and Retrieval-Clustering; E.1 [Data Structures]: —Graphs and networks

\section{General Terms}

Algorithms, Theory

\section{Keywords}

Biological networks, Dense module enumeration, K-medoids, Reverse search

\footnotetext{
Permission to make digital or hard copies of all or part of this work for personal or classroom use is granted without fee provided that copies are not made or distributed for profit or commercial advantage and that copies bear this notice and the full citation on the first page. Copyrights for components of this work owned by others than ACM must be honored. Abstracting with credit is permitted. To copy otherwise, or republish, to post on servers or to redistribute to lists, requires prior specific permission and/or a fee. Request permissions from permissions@acm.org. $B C B^{\prime} 16$, October 2-5, 2016, Seattle, WA, USA. Copyright 2016 ACM 978-1-4503-4225-4/16/10 ... \$15.00. http://dx.doi.org/10.1145/2975167.2985693.
}

\section{INTRODUCTION}

Tremendous amounts of data is getting generated in almost every field such as social networks and biological networks. Networks capture the interactions (connection) of entities. Researchers are often interested in finding closely connected group of nodes in a network such that the members of this group are highly related to each other. This problem to find closely connected and related group of nodes from a network/graph is also termed as dense subgraph mining.

Increasingly, several graph datasets have additional attributes which provide properties of either nodes or edges in the graph. For example, personal profile information such as age, interests, locale, etc are considered as node attribute data in a social network. Recently, the problem of mining dense subgraphs has been married with the concept of subspace similarity of node or edge attributes. Connected subgraphs which exhibit similarities over attribute data are also known as cohesive subgraphs or clusters. A lot of recent research has been focused in finding dense and cohesive subgraphs $[10,8,6]$, where the subgraphs are not only dense but are also 'similar' in a subset of attributes.

Cohesive dense subgraphs have many practical applications. Dense subgraph detection in protein-protein interaction (PPI) networks has already been shown to be useful in discovering protein function [2] and disease biomarkers [5, 4]. Finding closely related group of friends in social network can be used for recommendations or marketing of products.

The approach proposed in this paper uses a relaxed density definition as compared to some of the recent approaches such as, [10] which uses a quasi clique definition and is more restrictive. Also [8] employs nominal (integer values) attribute data while our approach is much broader and does not assume anything about the attribute data. In addition to the aforementioned papers another research paper [9] adapted the idea of reverse search in finding dense cohesive patterns. This paper further extends [9] by parallelizing the execution over multiple threads.

All of these algorithms require density and profile thresholds to prune the search space. For relaxed constraints a very high number of cohesive patterns are reported by these algorithms which overlap in both nodes and attributes. [9] provided an elegant solution to finding a representative set from this output space by finding the smallest dominating set. This paper introduces a novel application of K-medoids algorithm to find the representative set of cohesive patterns from the output space. 


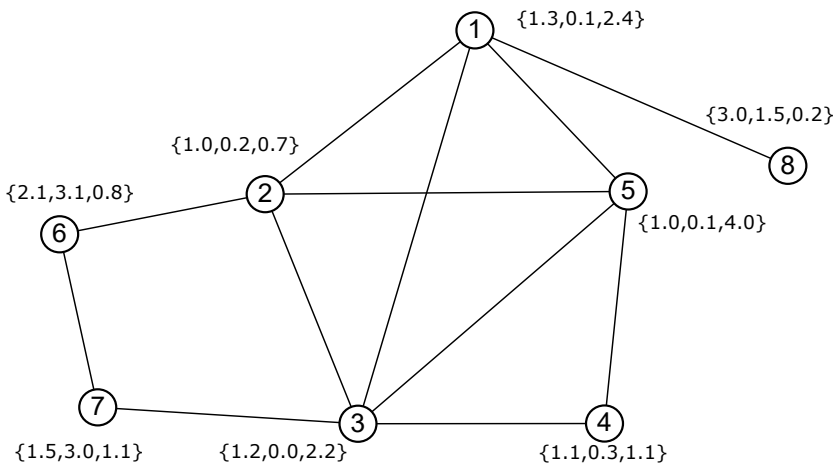

Figure 1 An example of an attributed graph.

We propose a multithreaded implementation to mine cohesive dense patterns from node attributed graphs. We apply the proposed algorithm on real world networks and compare the results. We also provide a novel application of K-medoids algorithm to find a representative set of patterns from output space.

The rest of the paper is organized as follows. The next section presents important definitions. In section 3, we discuss the multi threaded algorithm and the K-medoids algorithm in detail. Section 4 lists the experiment results on two real world data sets. Section 5 concludes the paper.

\section{PROBLEM DESCRIPTION}

This section introduces some definitions that are used throughout the paper. In this paper we consider a simple, undirected, connected, node attributed graph without self loops.

Let $G=(V, E, f)$ be an undirected graph, where $V=$ $\left\{v_{1}, \ldots, v_{n}\right\}$ is the set of vertices, $E \subseteq V \times V$ is the set of edges, and $f: V \rightarrow \mathbb{R}^{d}$ is an attribute function that maps a vertex to a $d$-dimensional real vector that represents the attributes for the vertex. An example of a graph with vertex attributes is shown in Figure 1 , where $V=$ $\{1,2,3,4,5,6,7,8\}$, and $E=\{(1,2),(1,3),(1,5), \cdots,(6,7)\}$

In this paper we intend to mine dense cohesive subgraphs. A subgraph is cohesive if its vertices are 'similar' in a subset of the attributes. We now provide details on attribute similarities and density.

For a subset of vertices $U \subseteq V$, we denote $G[U]=(U, E[U])$ as the subgraph of $G$ induced by $U$, i.e., $E[U]$ is the set of edges of $G$ whose endpoints are both in $U$.

The density of an induced subgraph $(U, E(U))$, denoted as $\rho$, is the ratio of the number of edges in the induced subgraph $(E[U])$ by the total possible edges in $G[U]$ :

$$
\rho(G[U])=\rho(U)=\frac{2|E[U]|}{|U|(|U|-1)}
$$

In Figure 1, for $U=\{1,2,3,4,5\}, \rho(U)=8 / 10=0.8$.

Definition 1. Given a tolerance threshold $t$ and a set of vertices $U$; where each vertex has $d$ dimensional vector representing attributes. The $k^{t h}$ attribute is considered a cohesive attribute for vertices in $U$ if the $k^{t h}$ attribute values for all vertices in $U$ differ by at most $t$.

$$
\forall u_{i}, u_{j} \in U:\left|f\left(u_{i}\right)[k]-f\left(u_{j}\right)[k]\right| \leq t
$$

For a threshold $t$, let $A(U, t)$ denotes the set of cohesive attributes, for simplicity we refer to $A(U, t)$ as $A(U)$ :

$$
A(U)=\left\{k_{1}, k_{2}, \cdots, k_{l}\right\}, 1 \leq k_{i} \leq d
$$

In Figure 1, for $U=\{3,4,5\}$ and $t=0.3, A(U)=\{1,2\}$ since the three vertices have 'similar' values in the $1^{\text {st }}$ and $2^{\text {nd }}$ attributes, i.e., the maximum difference between the attribute values for the three vertices in $U$ for each of the $1^{\text {st }}$ and $2^{\text {nd }}$ attribute is less than $t$.

Definition 2. Given a tolerance threshold $t$, a dimensionality threshold $s_{\min }$, an induced subgraph $G[U]$ is said to be a cohesive subgraph if the cardinality of the set of cohesive attributes is at least $s_{\text {min }}$, i.e., $|A(U)| \geq s_{\text {min }}$

The dimensionality threshold $s_{\min }$ is the minimum number of 'similar' attributes a set of vertices must have in order to form a cohesive subgraph. In Figure 1, for $t=0.3$ and $s_{\text {min }}=2$, the subgraph induced by $U=\{3,4,5\}$ is a cohesive subgraph.

Definition 3. Given a density threshold $\theta$, an attribute tolerance threshold $t$ and a dimensionality threshold $s_{\min }$ : $G[U]$ is a dense cohesive subgraph if it satisfies the following conditions.

1. The Density of the subgraph $G[U]$ is at least $\theta$, i.e., $\rho(U) \geq \theta$.

2. The number of relevant attributes should be at-least $s_{\text {min }}$, i.e., $|A(U)| \geq s_{\text {min }}$.

We can see from figure 1 that the subgraph induced by $U=\{1,2,3\}$ is both dense and cohesive for parameters $\left(\theta=0.8, t=0.3\right.$ and $\left.s_{\min }=2\right)$. The density $\rho(U)=\frac{3}{3}=1$ is greater than $(\theta=0.8)$ and vertices in $U$ have similar values in $1^{\text {st }}$ and $2^{\text {nd }}$ attributes $\left(s_{\min }=2\right)$ for $t=0.3$. A cohesive dense subgraph induced by $U$ is maximal if no superset $U^{\prime} \supseteq U$ is dense and cohesive. In this way we will not report every possible sub graph like $\{1,2,3\},\{1,2$, $5\}$ and $\{1,3,5\}$ which are subsets of the maximal cluster $\{1,2,3,4,5\}$ with same parameters $(\theta=0.8, t=0.3$ and $\left.s_{\min }=2\right)$.

Problem Definition: Given an attributed graph $G=$ $(V, E, f)$, three thresholds $\theta, t, s_{\text {min }}$, the problem of mining the set of maximal dense cohesive subgraphs is to find the set:

$$
\mathcal{P}=\left\{U_{1}, U_{2}, U_{3}, \cdots, U_{|\mathcal{P}|}\right\}
$$

such that every $U_{i} \in \mathcal{P}$ is a maximal dense cohesive subgraph. Each $U_{i}$ is a tuple $\left\{G_{i}, A_{i}\right\}$ containing a subgraph and its relevant attributes.

\section{METHODOLOGY}

In this section we introduce a multi-threaded implementation of RedCone [9] called MT_Redcone . Moreover, we propose a novel approach to find a representative set of maximal cohesive dense clusters from the output space of MT_Redcone .

RedCone mines maximal dense cohesive subgraphs by following a reverse search enumeration technique [1]. The reverse search technique guarantees that the enumeration of child search node is only dependent on its parent search 


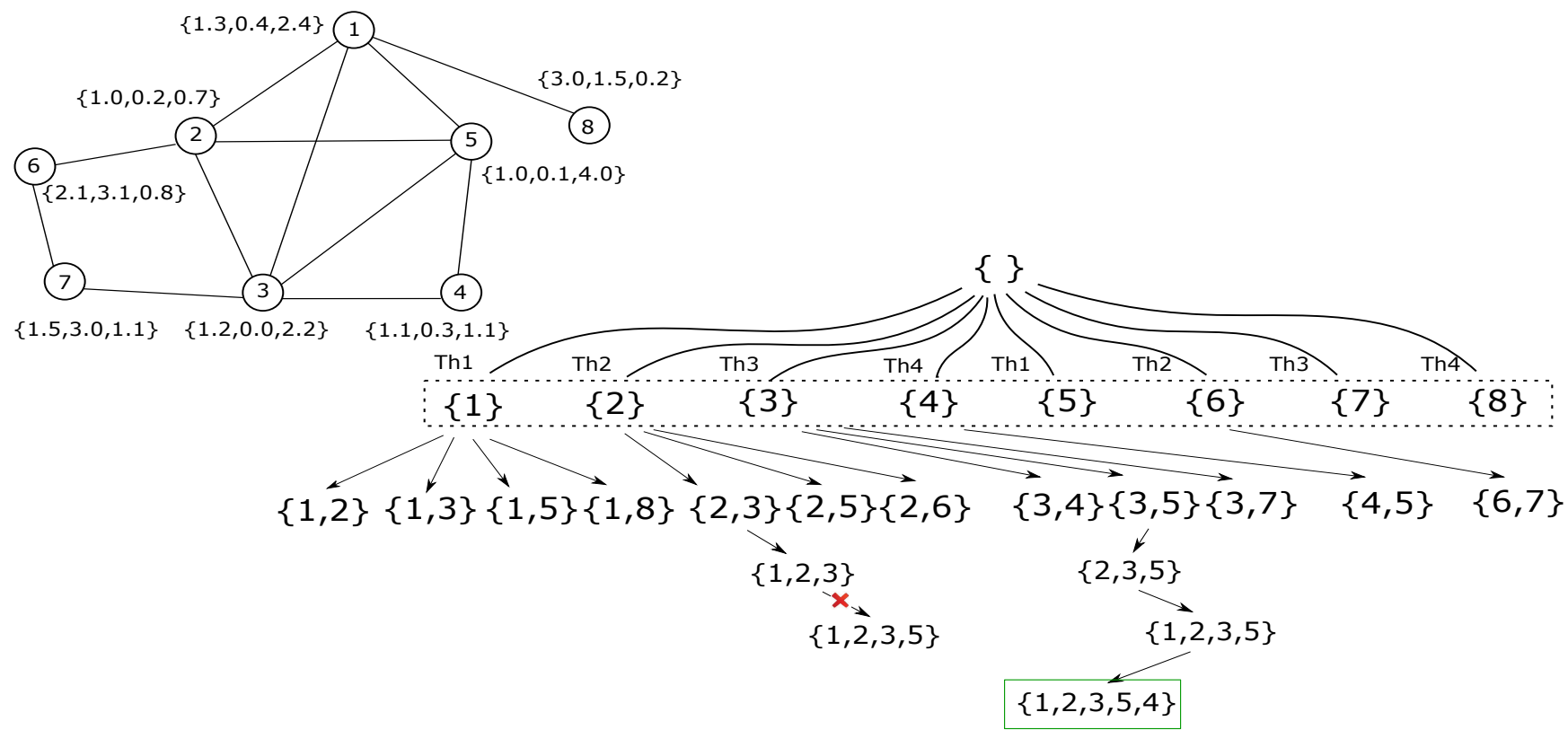

Figure 2 The input graph and its corresponding enumeration tree. There are four threads which build the enumeration tree independently. Each thread builds two subtrees from the first level children. Only a portion of the enumeration tree is shown for brevity. Crosses show which branches are pruned. The discovered maximal cluster are highlighted by a green box.

node and is independent of any shared structure. Figure 2 shows an example of the enumeration tree that MT_Redcone creates for the input graph shown in figure 1.

Utilizing this reverse search principle, the subtrees rooted under each first level node in the enumeration tree (figure 2) can be enumerated independently. This suggests that we can spawn multiple threads at the root, and each thread creates the sub tree under each of the first level nodes. Algorithm 1 shows the psuedo code for MT_Redcone . Apart from the usual inputs such as graph $G$, density threshold $\theta$, tolerance threshold $t$ and dimensionality threshold $s_{\text {min }}$, MT_Redcone also requires a number of threads input num $_{\text {threads. }}$. The algorithm begins by spawning the requested number of threads (line 3). Each thread then iterates over the first level nodes, selects a vertex and traverses its enumeration subtree (line 9). The output of this algorithm $\mathcal{P}$ is a list of maximal cohesive dense clusters.

These maximal cohesive dense clusters need to be further analyzed to derive useful insights and application. However, under relaxed density and cohesion constraints and a large input graph, the number of maximal dense cohesive clusters can become astronomically large [9, 10]. Moreover most of these output clusters have a high overlap in either the network structure or attribute data.

In the interest of making the analysis effective and efficient, a reduced set of these pattern is often sought after. We refer to the process of reducing the output maximal cohesive dense clusters to a smaller set as summarization. An effective mechanism to find a representative set of clusters from such output space has previously been discussed in [9].

A major limitation to the technique proposed in [9] is the construction of a similarity graph, which calculates the similarity between every pair of the output maximal cohesive dense cluster. This operation has a computational complexity of $O\left(n^{2}\right)$ where $n$ is the number of maximal cohesive dense clusters. The cost of constructing a similarity graph from an exponential output space makes this technique prohibitively restrictive. To address this issue, we propose a novel method of finding a representative set of clusters from a large output space. Another limitation of the technique in [9] is the requirement of a similarity threshold, which is used to remove edges with small weights (similarity) from the weighted similarity graph.

In this paper we map the problem of finding a reduced set of maximal cohesive dense clusters from a large output space to the problem of finding Kmedoids from a dataset. We treat the maximal cohesive dense patterns as objects and define a similarity (distance) function between these objects. We then run a modified version of K-medoids [14] clustering algorithm to find the $K$ medoids which are the most centrally located. Since the definition of distance is based on similarity instead of traditional distance (such as Euclidean distance), the most centrally located medoids are actually the most similar or representative cluster to all other cluster in the output space. We finally report these medoids as the representative maximal cohesive dense patterns.

The maximal cohesive dense patterns may overlap in either their network structure or attributes. Hence our definition of distance is based on the similarity in the network structure and the relevant attributes.

Given two patterns, $U, U^{\prime}$, let $S_{U U^{\prime}}$ denotes the vertex similarity which is defined as the Jaccard similarity coefficient between the sets of vertices of the two clusters.

$$
S_{U U^{\prime}}^{v}=\frac{\left|U \cap U^{\prime}\right|}{\left|U \cup U^{\prime}\right|}
$$

Moreover, the attribute similarity between the two patterns is captured by the Jaccard similarity coefficient be- 
$\overline{\text { Algorithm } 1 \text { Pseudo-code for a parallel (Multithreaded) }}$ algorithm for Maximal Cohesive Dense Cluster Discovery

\section{Input:}

$G=(V, E, f)$ : an attributed graph

min_size: the minimum size of cluster to include in results $\theta$ : density threshold

$t$ : tolerance threshold between two attribute values in a single subspace

$s_{\text {min }}$ : minimum number of similar attributes per cluster num $_{\text {threads }}$ : Number of threads to spawn for parallel execution

\section{Output:}

$\mathcal{P}$ : maximal cohesive dense clusters

1: Remove all non cohesive edges from input graph

2: $\mathcal{P}=\{\}$

3: threads []$=$ spawn_threads $\left(\right.$ num $\left._{\text {threads }}\right)$

4: start_all_threads(threads[],ThreadStart)

5: join_all_threads(threads[])

6: function THREADSTART

7: while there are more vertices do

8: $\quad$ Ensuring mutual exclusion, choose a vertex, $v$

9: $\quad$ execute_thread(Mineclusters $(v))$

10: end while

11: end function

12: function Mineclusters $(U)$

13: $\quad$ locally_maximal $\leftarrow$ true

14: $\quad$ for $v \in V \backslash U$ do

15: $\quad$ Let $U^{\prime}=U \cup v$

16: $\quad$ if $\rho\left(U^{\prime}\right) \geq \theta$ and $\left|A\left(U^{\prime}\right)\right| \geq s_{\text {min }}$ then

17: $\quad$ locally_maximal $\leftarrow$ false

18: $\quad$ if $\operatorname{IsChILD}\left(U^{\prime}, U\right)$ then

19: $\quad \operatorname{MineClusters}\left(U^{\prime}\right)$

20: $\quad$ end if

21: $\quad$ end if

22: $\quad$ end for

23: $\quad$ if locally_maximal and $|U| \geq$ min_size then

24: $\quad \mathcal{P}=\mathcal{P} \cup U$

25: $\quad$ end if

26: end function

27: return $\mathcal{P}$

tween the sets of relevant attributes,

$$
S_{U U^{\prime}}^{a}=\frac{\left|A(U) \cap A\left(U^{\prime}\right)\right|}{\left|A(U) \cup A\left(U^{\prime}\right)\right|}
$$

Next, we define the pattern similarity as linear combination of the vertex and attribute similarities as follows:

$$
S_{U U^{\prime}}=\alpha * S_{U U^{\prime}}^{v}+(1-\alpha) * S_{U U^{\prime}}^{a}
$$

where $\alpha$ is a user-defined parameter to control the contribution of the vertex similarity to the total pattern similarity.

Figure 3 shows the Kmedoids algorithm on a set of maximal cohesive dense clusters $\left\{p_{1}, p_{2}, \ldots, p_{5}\right\}$. Figure 3(a) shows the maximal cohesive dense patterns and also lists the cohesive attributes of each pattern. The algorithms starts by selecting $K$ random patterns as the initial medoids.

After the random selection of the initial medoids, the algorithm assigns each pattern to its most similar medoid. Consequently a set of patterns are formed for each medoid containing the medoid itself, and all the patterns assigned to it, as shown in figure 3 (c). Next, new medoids are recalculated for each partition, as shown in figure $3(\mathrm{~d})$. The process of assigning patterns to medoids and recalculating the medoids is repeated until a steady state is reached, i.e., new medoids are exactly same as the previous medoids or a maximum number of iterations has been reached. The final medoids at the steady state are the representative maximal cohesive dense clusters.

Given a set of patterns $\left\{p_{1}, p_{2}, \ldots, p_{n}\right\}$, their $K$ partitions $\left\{c_{1}, c_{2}, \ldots, c_{K}\right\}$ and their respective medoids $\left\{m_{1}, m_{2}, \ldots, m_{K}\right\}$, the modified K-medoids algorithm, tries to maximize the similarity between patterns, $p_{i}$, and their medoids, $m_{i}$.

$$
\sum_{i=1}^{K} \sum_{p \in c_{i}} S_{p m_{i}}
$$

where $S$ represents the similarity between two maximal cohesive dense patterns.

Now we shall define some metrics to understand the quality of these output medoids (representative clusters) as produced by the K-medoids algorithm.

For a clustering $\left\{c_{1}, c_{2}, \ldots, c_{K}\right\}$ and their respective medoids $\left\{m_{1}, m_{2}, \ldots, m_{K}\right\}$, we define the average intra partition similarity as the average sum of the similarities between patterns and their corresponding corresponding medoid.

$$
\text { AvgPartition Sim }=\frac{\sum_{i=1}^{K} \sum_{p \in c_{i}} S_{p m_{i}}}{n}
$$

The AvgPartitionSim similarity captures the quality of all clustering. A higher value indicates that medoids are more similar to their constituent patterns, suggesting a better partitioning.

We next define the average inter medoid similarity as average of sum of the pair wise similarities between the set of medoids.

$$
\text { AvgMedoidSim }=\frac{\sum_{i=1}^{K} \sum_{j=i+1}^{K} S_{m_{i} m_{j}}}{K *(K-1) / 2}
$$

As opposed to the AvgPartitionSim, AvgMedoidSim indicates the dissimilarity between the detected medoids. If the medoids are sufficiently similar to all points in their partition than they should be dissimilar to other medoids. In high quality partitions, we expect a high value for AvgPartitionSim and a low value for AvgMedoidSim indicating dissimilarity with other medoids.

Finally, the complexity of each iteration of K-medoids is $O\left(K *(n-K)^{2}\right)$, where $K$ represents the desired number of representative clusters [16].

\section{EXPERIMENTAL RESULTS}

This section outlines the execution of the proposed algorithm on two real-world protein-protein interaction networks and associated attribute data.

1. Yeast: We use the Yeast protein-protein interaction network from the Biological General Repository for Interaction Datasets (BioGRID) [15]. The Yeast interaction network has 6, 249 vertices and 224, 587 edges. Gene profile attribute information correspond to the differential expression value of each gene when exposed to 173 different experiments [7]. Each gene has 173 real attributes.

2. Human: We use the Human protein-protein interaction network from the BioGRID [15]. The network has 20,313 vertices and 230, 845 interactions. For attribute data, we use the dysregulation profile of genes 


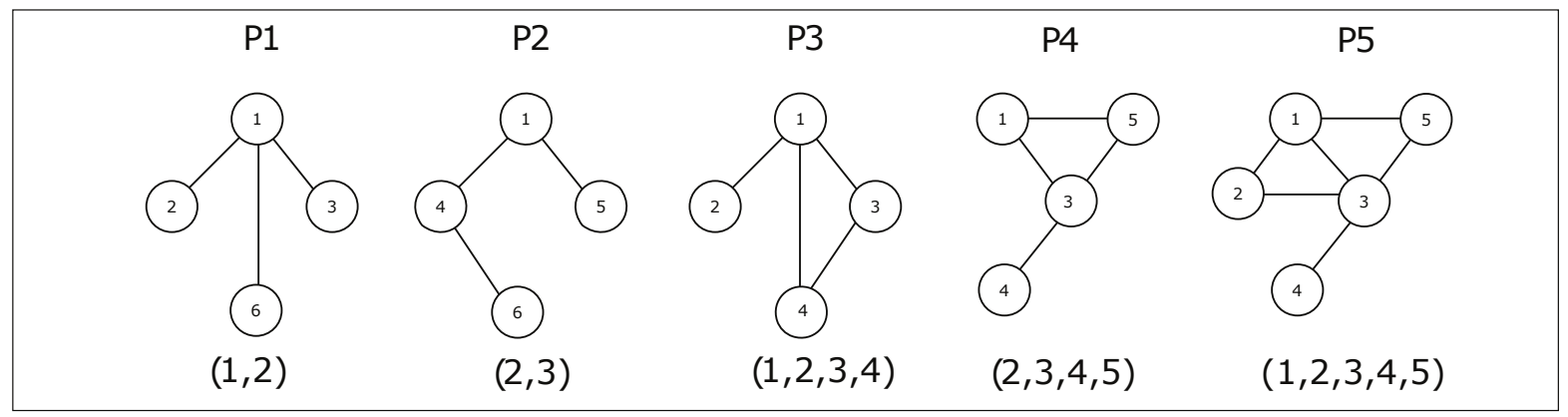

(a)

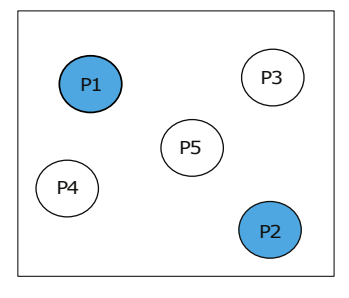

(b)

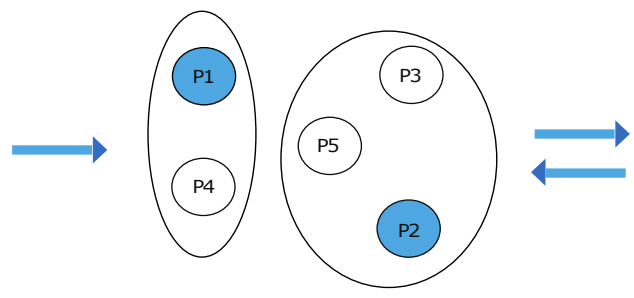

(c)

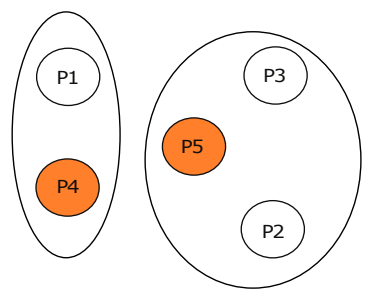

(d)

Figure 3 Representative clusters. (a) Set of maximal cohesive dense clusters, (b) Projecting maximal cohesive dense clusters to points in space and selecting $\mathrm{K}$ random medoids. (c) Finding partitions around previously selected medoids $(P 1$ and $P 2)$. (d) Calculating true medoids $m 1=P 4$ and $m 2=P 5$ for each partition. The algorithm alternates between step (c) and (d) till a steady state is reached. The set of steady state medoids $m 1, m 2$ are the representative maximal cohesive dense clusters.

in 13 different cancers (attributes) where ' 1 ' indicates that the gene is dysregulated [13].

The multithreaded algorithm is implemented in $\mathrm{C}++$ and all experiments were run independently on machine running Ubuntu Linux operating system with an Intel Xeon $(3.3 \mathrm{GHz})$ processor, with 8 cores and 16 Gigabytes of main memory.

\subsection{Topological Attributes of Dense Cohesive Patterns}

The topological properties of the reported maximal dense cohesive patterns for varying density and cohesive constraints are shown in Table 1 and 2 for Yeast and Human datasets respectively. In these tables, $|N|$ denotes the number of maximal cohesive dense patterns and $\bar{N}$ represents the average size.

As the attribute cohesion constraint get relaxed, the number of cohesive patterns increases; the same trend is observed when the density threshold is relaxed.

\subsection{Runtime}

In this section, we compare the runtime of MT_Redcone with varying number of threads. Figures 4 and 5 plots the runtime for varying values of density $(\theta)$ and dimension $\left(S_{\min }\right)$, respectively, for the Yeast dataset. Figures 6 and 7 plots the runtime for varying values of density $(\theta)$ and dimension $\left(S_{\min }\right)$, respectively, for the Human dataset. We ran multiple experiments with varying number of threads, beginning from a single thread (RedCone) to parallelizing with upto 32 threads. MT_Redcone is multiple times faster
Table 1 Topological properties of maximal cohesive dense patterns for the Yeast dataset.

\begin{tabular}{|ccc|cc|}
\hline \multicolumn{3}{|c|}{ Parameters } & \multicolumn{3}{c|}{} \\
$\theta / \gamma$ & $t$ & $s_{\min }$ & $|N|$ & $\bar{N}$ \\
\hline \hline 0.6 & 0.2 & 60 & 1769 & 4.60 \\
0.6 & 0.2 & 75 & 572 & 4.59 \\
0.6 & 0.3 & 60 & 49465 & 4.81 \\
0.6 & 0.3 & 75 & 8022 & 4.80 \\
\hline 0.7 & 0.2 & 60 & 187 & 4.86 \\
0.7 & 0.2 & 75 & 63 & 4.97 \\
0.7 & 0.3 & 60 & 8250 & 4.59 \\
0.7 & 0.3 & 75 & 1005 & 4.82 \\
\hline 0.8 & 0.2 & 60 & 108 & 4.20 \\
0.8 & 0.2 & 75 & 31 & 4.39 \\
0.8 & 0.3 & 60 & 5340 & 4.08 \\
0.8 & 0.3 & 75 & 525 & 4.11 \\
\hline 0.9 & 0.2 & 60 & 10 & 4.30 \\
0.9 & 0.2 & 75 & 4 & 4.75 \\
0.9 & 0.3 & 60 & 1654 & 4.06 \\
0.9 & 0.3 & 75 & 77 & 4.12 \\
\hline
\end{tabular}

than the single thread execution of RedCone. The speedup is bounded by the number of cores in the CPU which is 8 in our machine. Moreover, as we increase the number of threads beyond 8, no gain in speedup is obtained and we start seeing the impact of the computational overhead (this behavior can be seen in figure 4).

\subsection{Representative set}

In this section we discuss our results from the summarization process. As noted previously, finding a reduced 


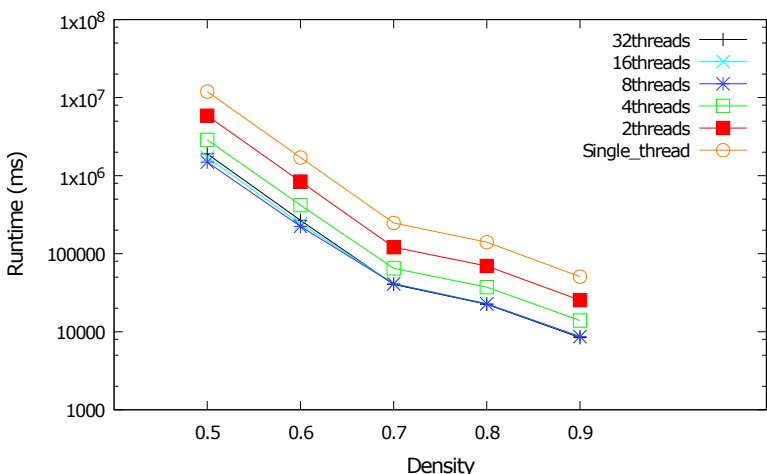

(a)

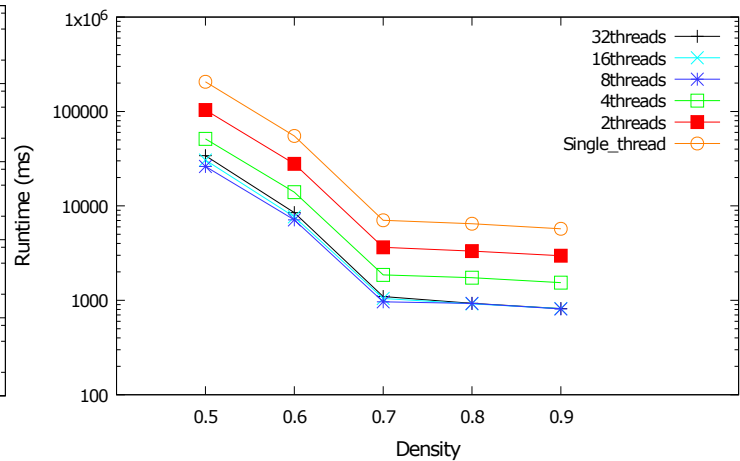

(b)

Figure 4 Runtime comparison of multiple threads on the Yeast dataset with varying density, parameters : (a) $t=0.4$ and $s_{\text {min }}=60(\mathrm{~b}) t=0.3$ and $s_{\min }=65$

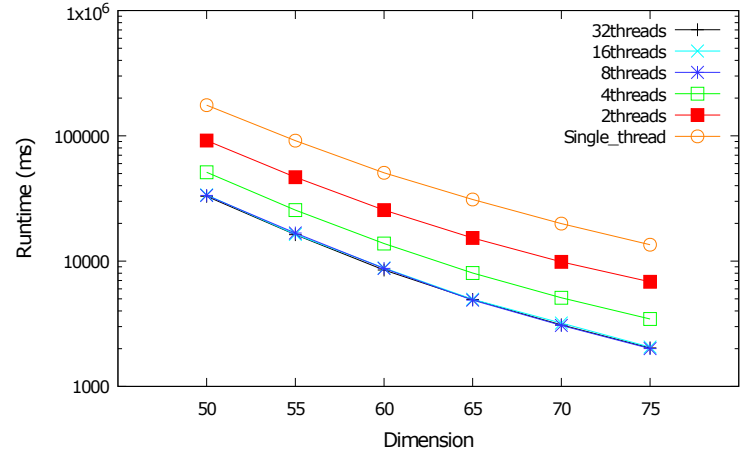

(a)

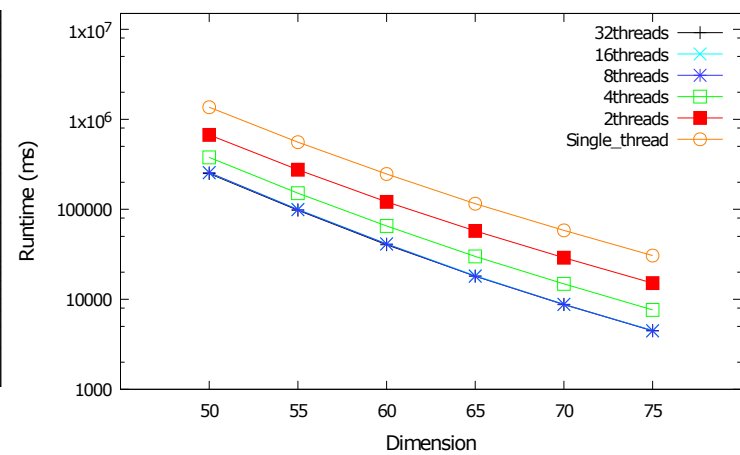

(b)

Figure 5 Runtime comparison of multiple threads on the Yeast dataset with varying dimension, parameters : (a) $t=0.4$ and $\theta=0.9$ (b) $t=0.4$ and $\theta=0.7$

Table 2 Topological properties of maximal cohesive dense patterns for the Human dataset.

\begin{tabular}{|c|c|c|c|}
\hline $\begin{array}{l}\text { Par } \\
\theta / \gamma\end{array}$ & $\begin{array}{l}\text { heters } \\
s_{\min }\end{array}$ & $|N|$ & $\bar{N}$ \\
\hline 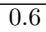 & 2 & $\overline{585588}$ & 6.66 \\
\hline 0.6 & 3 & 165668 & 7.10 \\
\hline 0.6 & 4 & 68441 & 7.22 \\
\hline 0.6 & 5 & 29374 & 7.30 \\
\hline 0.7 & 2 & 47077 & 5.39 \\
\hline 0.7 & 3 & 16178 & 5.60 \\
\hline 0.7 & 4 & 8084 & 5.62 \\
\hline 0.7 & 5 & 4036 & 5.69 \\
\hline 0.8 & 2 & 5842 & 5.30 \\
\hline 0.8 & 3 & 2117 & 5.31 \\
\hline 0.8 & 4 & 1158 & 5.41 \\
\hline 0.8 & 5 & 623 & 5.21 \\
\hline 0.9 & 2 & 864 & 5.19 \\
\hline 0.9 & 3 & 377 & 5.26 \\
\hline 0.9 & 4 & 235 & 5.02 \\
\hline 0.9 & 5 & 107 & 4.99 \\
\hline
\end{tabular}

representative set is important when there are millions of maximal cohesive dense patterns. A reduced representative set (of maximal cohesive dense clusters) is more manageable and is better suited for analysis as they closely represent the entire population of output clusters.

For summarization we used the the High Confidence Yeast

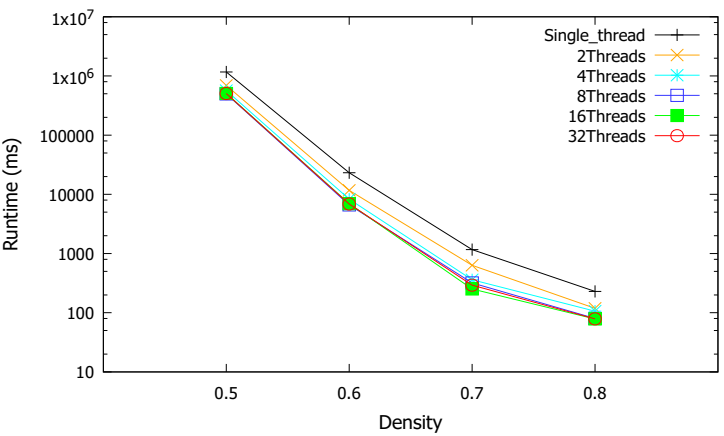

Figure 6 Runtime comparison of multiple threads on the Human dataset with varying density, parameters : $s_{\text {min }}=4$

(YeastHC) interaction network [3]. This network has 4008 vertices and 9857 edges. Similar to the Yeast dataset, we used attribute values obtained from gene profile information from 173 experiments. We ran a modified K-medoids algorithm on the output space of MT_Redcone for varying parameters of both MT_Redcone and K-medoids . Table 3 presents some of the results of K-medoids algorithm. $\theta$, $\gamma, t, s_{\min }$ represent the parameters for the MT_Redcone and $|N|$ denotes the number of output maximal cohesive dense clusters. $K$ and $\alpha$ are the parameters for K-medoids 


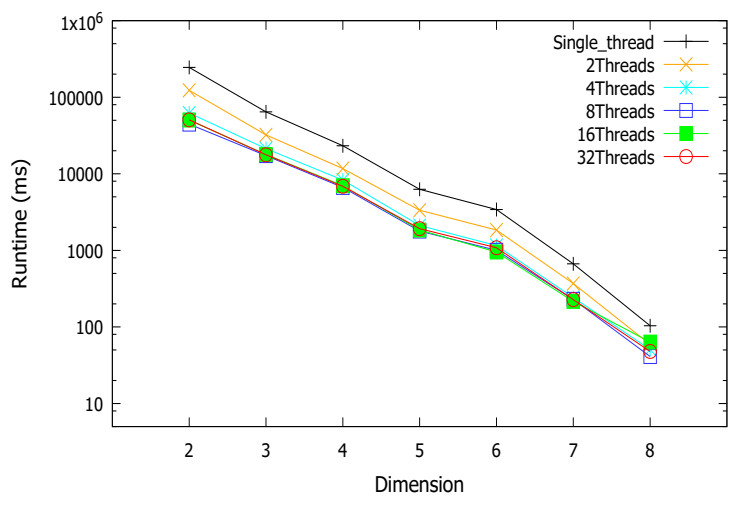

Figure 7 Runtime comparison of multiple threads on the Human dataset with varying dimension, parameters : $\theta=$ 0.6

Table 3 Similarities of medoids in Representative Set.

\begin{tabular}{|cccc|cc|cc|}
\hline \multicolumn{4}{|c|}{ MT_Redcone } & \multicolumn{2}{|c|}{ K-medoids } & \multicolumn{3}{|c|}{ Similarities } \\
$\theta / \gamma$ & $t$ & $s_{\min }$ & $|N|$ & $K \%$ & $\alpha$ & AvgPartitionSim & AvgMedoidSim \\
\hline \hline 0.5 & 0.2 & 15 & 996 & $10 \%$ & 0.5 & 0.53 & 0.053 \\
0.7 & 0.2 & 15 & 2417 & $10 \%$ & 0.5 & 0.48 & 0.085 \\
0.8 & 0.2 & 10 & 6100 & $10 \%$ & 0.5 & 0.50 & 0.038 \\
0.9 & 0.3 & 15 & 9989 & $10 \%$ & 0.5 & 0.55 & 0.059 \\
\hline 0.5 & 0.2 & 15 & 996 & $70 \%$ & 0.5 & 0.88 & 0.052 \\
0.7 & 0.2 & 15 & 2417 & $70 \%$ & 0.5 & 0.86 & 0.077 \\
0.8 & 0.2 & 10 & 6100 & $70 \%$ & 0.5 & 0.86 & 0.038 \\
0.9 & 0.3 & 15 & 9989 & $70 \%$ & 0.5 & 0.87 & 0.059 \\
\hline 0.5 & 0.2 & 15 & 996 & $10 \%$ & 0.9 & 0.58 & 0.024 \\
0.7 & 0.2 & 15 & 2417 & $10 \%$ & 0.9 & 0.53 & 0.007 \\
0.8 & 0.2 & 10 & 6100 & $10 \%$ & 0.9 & 0.57 & 0.022 \\
0.9 & 0.3 & 15 & 9989 & $10 \%$ & 0.9 & 0.61 & 0.031 \\
\hline
\end{tabular}

where $K$ represents the number of representative patterns and $\alpha$ is a user defined parameter to control the similarity between clusters. $K \%$ is the percentage of patterns. We ran multiple experiments varying both sets of parameters and calculated the average intra partition similarity and average inter medoid similarity as defined previously. Figure 8 and 9 plot the average intra partition similarity and average inter medoid similarity for varying values of $\alpha$ and $K$.

As $\alpha$ increases, the pattern similarity is biased towards the network structure similarity. In figure 8 , notice as $\alpha$ is increasing the AvgPartitionSim (average intra partition similarity) is increasing and AvgMedoidSim (average inter medoid similarity) is decreasing. Recall that high values of AvgPartitionSim and low values of AvgMedoidSim suggests good partitioning. In other words, the maximal cohesive dense clusters resulting from this YeastHC dataset has more similarity in its network structure over their attributes. This trait is demonstrated in figure 8, as increasing $\alpha$ values increases similarity among these clusters and hence form better partitions.

In figure 9, we observe the trend in AvgPartitionSim and AvgMedoidSim values with increasing the desired number of representative clusters. As $K$ increases, we increase the number of partitions in the output space. We see a steady increase in AvgPartitionSim while AvgMedoidSim is pretty much flat. This is expected because with increasing number of partitions the members in each partition decrease, and, the similarity of each member in the

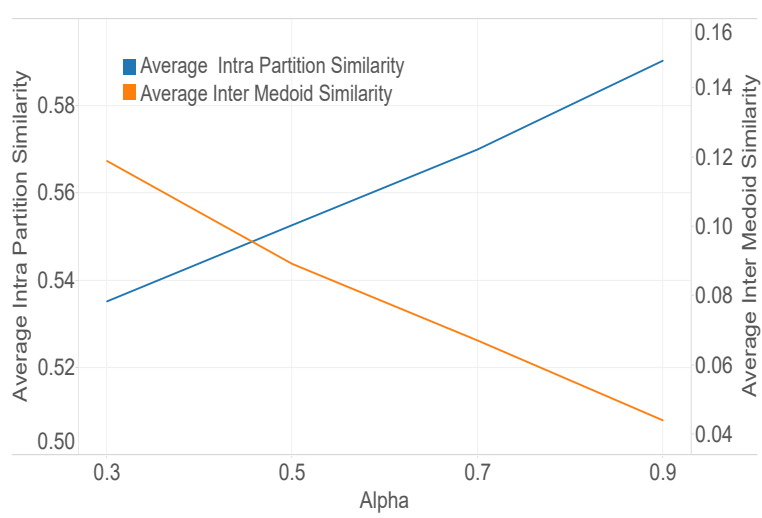

Figure $\quad 8 \quad$ Average intra partition similarity AvgPartitionSim and average inter medoid similarity AvgMedoidSim with varying alpha values.

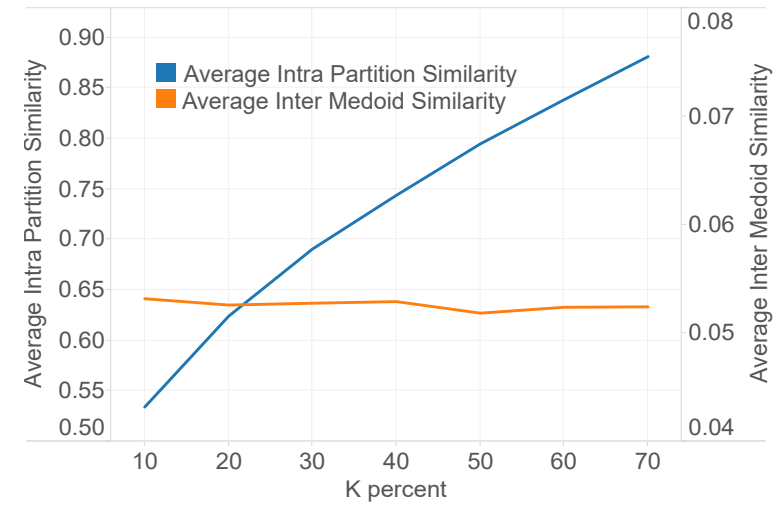

Figure 9 Average intra partition similarity AvgPartitionSim and average inter medoid similarity AvgMedoidSim with varying K values.

partition to their respective medoid increases. This increase in similarity is captured by the increasing trend of AvgPartitionSim. At $100 \%$ value of $K$, each cluster is its own partition and medoid and has a perfect similarity score of 1 .

\subsection{Biological Analysis}

We performed biological enrichment analysis of the reported patterns for the Human dataset using the Database for Annotation, Visualization, and Integrated Discovery, DAVID [12, 11]. To assess the biological relevance of the maximal cohesive patterns, we attempted to find enrichment (over-representation) of KEGG pathways in the patterns. Figure 10 shows that a large percentage $(80 \%$ to $100 \%$ ) of the reported patterns are enriched with known KEGG pathways. Moreover, dense modules whose genes are dysregulated in more diseases are more likely to be enriched with KEGG pathways. For Example, for density = 0.8 , and $s_{\min }=4,82 \%$ percent of the patterns are enriched with at least one KEGG pathway, while for $s_{\min }=$ 8 , the percentage is $100 \%$. A similar trend is observed for other density thresholds. For density $=0.9$, and $s_{\min }=$ 6, 42 modules (out of 67) are enriched. Examples of enriched KEGG pathways include hsa04115 ('p53 signaling pathway'), hsa05212 ('Pancreatic cancer'), hsa05206 ('Mi- 
croRNAs in cancer'), hsa05222 ('Small cell lung cancer'), and hsa05215 (Prostate cancer).

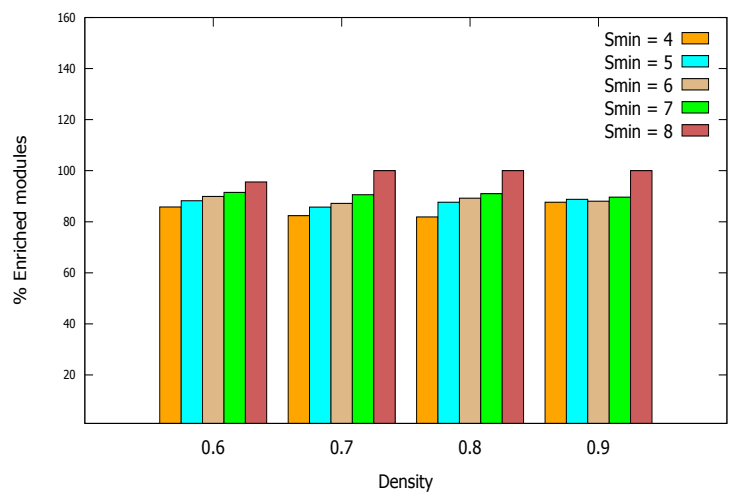

Figure 10 KEGG pathway analysis for modules reported by Human dataset with varying density and attribute parameters

\section{CONCLUSION}

We proposed a multithreaded algorithm for mining the set of maximal cohesive subnetworks from a graph with node attributes. To address the information overload resulting from the large number of mined patterns due to the combinatorial nature of the problem, we proposed a Kmedoidsbased algorithm for selected $K$ representative patterns. Experiments on real biological networks and gene expression attributes show that the multithreaded algorithm achieves a runtime performance increase. Moreover, biological enrichment analysis show that the maximal cohesive patterns are biologically relevant.

\section{ACKNOWLEDGMENTS}

This publication was made possible by National Science Foundation (NSF) grant IIS-1423321.

\section{REFERENCES}

[1] D. Avis and K. Fukuda. Reverse search for enumeration. Discrete Applied Mathematics, 65(1):21-46, 1996.

[2] S. Bandyopadhyay, R. Sharan, and T. Ideker. Systematic identification of functional orthologs based on protein network comparison. Genome research, 16(3):428-435, 2006.

[3] N. N. Batada, T. Reguly, A. Breitkreutz, L. Boucher, B.-J. Breitkreutz, L. D. Hurst, and M. Tyers. Still stratus not altocumulus: further evidence against the date/party hub distinction. PLoS biology, 5(6):e154, 2007.

[4] S. A. Chowdhury, R. K. Nibbe, M. R. Chance, and M. Koyutürk. Subnetwork state functions define dysregulated subnetworks in cancer. Journal of Computational Biology, 18(3):263-281, 2011.

[5] H.-Y. Chuang, E. Lee, Y.-T. Liu, D. Lee, and T. Ideker. Network-based classification of breast cancer metastasis. Molecular systems biology, 3(1), 2007.
[6] R. Colak, F. Moser, J. S.-C. Chu, A. Schönhuth, N. Chen, and M. Ester. Module discovery by exhaustive search for densely connected, co-expressed regions in biomolecular interaction networks. PloS one, 5(10):e13348, 2010.

[7] A. P. Gasch, P. T. Spellman, C. M. Kao, O. Carmel-Harel, M. B. Eisen, G. Storz, D. Botstein, and P. O. Brown. Genomic expression programs in the response of yeast cells to environmental changes. Molecular biology of the cell, 11(12):4241-4257, 2000.

[8] E. Georgii, S. Dietmann, T. Uno, P. Pagel, and K. Tsuda. Enumeration of condition-dependent dense modules in protein interaction networks. Bioinformatics, 25(7):933-940, 2009.

[9] A. Goparaju, T. Brazier, and S. Salem. Mining representative maximal dense cohesive subnetworks. Network Modeling Analysis in Health Informatics and Bioinformatics, 4(1):1-11, 2015.

[10] S. Gunnemann, I. Farber, B. Boden, and T. Seidl. Subspace clustering meets dense subgraph mining: A synthesis of two paradigms. In Data Mining (ICDM), 2010 IEEE 10th International Conference on, pages 845-850. IEEE, 2010.

[11] D. W. Huang, B. T. Sherman, and R. A. Lempicki. Bioinformatics enrichment tools: paths toward the comprehensive functional analysis of large gene lists. Nucleic Acids Res., 37(1):1-13, 2009.

[12] D. W. Huang, B. T. Sherman, and R. A. Lempicki. Systematic and integrative analysis of large gene lists using david bioinformatics resources. Nature Protoc., 4(1):44-57, 2009.

[13] W. Jiang, R. Mitra, C.-C. Lin, Q. Wang, F. Cheng, and Z. Zhao. Systematic dissection of dysregulated transcription factor-mirna feed-forward loops across tumor types. Briefings in bioinformatics, page bbv107, 2015.

[14] L. Kaufman and P. Rousseeuw. Clustering by means of medoids. Statistical Data Analysis Based on the L1-Norm and Related Methods, pages 405-416, 1987.

[15] T. K. Prasad, R. Goel, K. Kandasamy, S. Keerthikumar, S. Kumar, S. Mathivanan, D. Telikicherla, R. Raju, B. Shafreen, A. Venugopal, et al. Human protein reference databaseâĂT $\breve{T} 2009$ update. Nucleic acids research, 37(suppl 1):D767-D772, 2009.

[16] A. P. Reynolds, G. Richards, B. de la Iglesia, and V. J. Rayward-Smith. Clustering rules: A comparison of partitioning and hierarchical clustering algorithms. Journal of Mathematical Modelling and Algorithms, 5(4):475-504, 2006. 